\title{
Summary of session C1: pulsar timing arrays
}

\author{
R. M. Shannon - S. Chamberlin - N. J. Cornish • J. A. Ellis • \\ C. M. F. Mingarelli - D. Perrodin - P. Rosado - A. Sesana - S. R. Taylor • \\ L. Wen - C. G. Bassa - J. Gair - G. H. Janssen - R. Karuppusamy • \\ M. Kramer • K. J. Lee • K. Liu • I. Mandel • M. Purver • T. Sidery • \\ R. Smits - B. W. Stappers - A. Vecchio
}

Received: 31 December 2013 / Accepted: 19 June 2014 / Published online: 9 July 2014

(C) Springer Science+Business Media New York 2014

\begin{abstract}
This paper summarizes parallel session C1: Pulsar Timing Arrays of the Amaldi10/GR20 Meeting held in Warsaw, Poland in July 2013. The session showcased recent results from pulsar timing array collaborations, advances in modelling the gravitational-wave signal, and new methods to search for and characterize gravitational waves in pulsar timing array observations.
\end{abstract}

This article belongs to the Topical Collection: The First Century of General Relativity: GR20/Amaldi10.

\author{
R. M. Shannon ( $\square)$ \\ CSIRO Astronomy and Space Science, Epping, Australia \\ e-mail: ryan.shannon@csiro.au \\ S. Chamberlin · J. A. Ellis \\ University of Wisconsin, Milwaukee, WI, USA \\ N. J. Cornish \\ Montana State University, Bozeman, MT, USA \\ C. M. F. Mingarelli · I. Mandel - T. Sidery · A. Vecchio \\ University of Birmingham, Birmingham, UK \\ D. Perrodin \\ Osservatorio Astronomico di Cagliari, Cagliari, Italy \\ P. Rosado - A. Sesana \\ Max Planck Institute for Gravitational Physics, \\ Albert Einstein Institute, Potsdam, Germany \\ J. Gair · S. R. Taylor \\ University of Cambridge, Cambridge, UK \\ L. Wen \\ University of Western Australia, Crawley, Australia
}


Keywords Gravitational waves · Pulsars

\section{Introduction}

Radiofrequency observations of millisecond pulsars provide one potential avenue for peering into the gravitational-wave universe. Pulsars are rapidly rotating neutron stars known for their rotational stability. If the misaligned magnetic axis of the star crosses our line of sight, a pulse of radio emission is observed. The pulses can be time-tagged to a precision of $\sim 30 \mathrm{~ns}$ and therefore are sensitive to many effects that can cause variations in pulse arrival times.

Gravitational waves (GWs) passing the pulsar-Earth line-of-sight cause one such variation. By studying the correlated response to an ensemble of millisecond pulsars (commonly referred to as a pulsar timing array, PTA) it is possible to distinguish the effects of GWs, which produce a quadrupolar correlation in TOA variations, from other effects, such as intrinsic (and uncorrelated between the pulsars) spin noise, uncertainties in the solar system ephemeris (dipolar correlation), and terrestrial time variations, which induce a monopolar correlation. In parallel session $\mathrm{C} 1$, progress on all aspects of PTA research were highlighted.

The strongest source of gravitational waves expected in the PTA band (GW frequencies from $10 \mu \mathrm{Hz}$ to $1 \mathrm{nHz}$ ) are associated with binary supermassive black hole binaries (SMBHBs) in the throes of merger. SMBHBs can produce different classes of GW emission: individual sources can be identified if they are is sufficiently strong emitters of GWs. Additionally, a stochastic gravitational-wave background (GWB) is produced from the superposition of all of the sources. In this session, Sesana describes recent models for the signal based on recent observational and simulation models of galaxy black hole co-evolution. Rosado describes a method to simulate a GWB by populating Sloan Digital Sky Survey galaxies with SMBHBs.

Current observational efforts are found at all of the most sensitive radio telescopes in the world. Presentations by Perrodin and Shannon provide updates from the European Pulsar Timing Array (EPTA) and Parkes Pulsar Timing Array (PPTA). The third major effort, based in North America, (North American Nanohertz Observatory for Gravitational Waves, NANOGrav), is summarized by M. McLaughlin elsewhere in the proceedings for the meeting.

C. G. Bassa - G. H. Janssen · M. Purver · B. W. Stappers

Jodrell Bank Centre for Astrophysics, University of Manchester,

Manchester, UK

R. Karuppusamy · K. J. Lee · M. Kramer

Max Planck Institute for Radio Astronomy,

Bonn, Germany

K. Liu

CNRS, Nançay Observatory,

Orleans, France

R. Smits

ASTRON, Dwingeloo, The Netherlands 
As the field advances, the techniques used to detect the GWB are becoming more refined. Chamberlin describes an effort to develop a pipeline to search for GWs in PTA datasets. At the same time, questions are arising as to the nature of the stochasticity of the background. Mingarelli and Taylor describe methods to search for and measure the anisotropy of the background.

If the background is particularly non-Gaussian, or there is a massive black hole binary lurking nearby, it is possible that GWs from a single source will be the first evidence of our GW universe. Ellis, Wen, and Cornish describe describe methods for searching for and localising single sources.

\section{Probing massive black hole binaries with pulsar timing arrays (Presenter: A. Sesana)}

Inspiralling SMBHBs populating merging galaxies throughout the Universe are expected to generate the dominant signal in the PTA band (see, e.g., [1]). The first systematic investigation of the range of GW signal amplitudes consistent with observationally based estimates of the SMBHB assembly in the low redshift Universe was recently conducted [2]. As the expected GW signal scales as $\propto f^{-2 / 3}$, it is customary to parametrize it as

$$
h_{c}(f)=A\left(\frac{f}{\text { year }^{-1}}\right)^{-2 / 3},
$$

where $A$ is a model dependent constant that represents the amplitude of the signal at the reference frequency $f=1$ year $^{-1}$. Figure 1 show the distribution of $A$ predicted by those empirical models. The $90 \%$ confidence interval of the expected signal is centered around $10^{-15}$, spanning about an order of magnitude. Uncertainties are mostly related to our poor knowledge of the galaxy and SMBHB merger rate and of the supermassive black hole (SMBH)-host relations (i.e., the $M_{\mathrm{BH}}-\sigma$ or the $M_{\mathrm{BH}}-M_{\text {bulge }}$ relations, see [2] for details). As an example, the $\mathrm{SMBH}$-host relations were recently updated (see, e.g., [3]) to include measurements of overmassive SMBHs in brightest cluster galaxies (BCGs) [4]. The subset of models employing those new relations predicts a $\mathrm{GW}$ signal which is a factor of $\approx 2$ larger than previously expected. The current best published limit [5] starts to dig significantly into the expected signal range, indicating that PTAs will possibly provide a detection within the next decade, or it will allow us to place strong limits on the cosmological population of SMBHBs at low redshift, which are among the most elusive, yet fundamental, building blocks of our Universe.

\section{Gravitational wave detection with the large European array for pulsars (Presenter: D. Perrodin)}

The European Pulsar Timing Array (EPTA), which is part of the International Pulsar Timing Array (IPTA), is a collaboration of pulsar scientists working with five large European radio telescopes (Effelsberg, Westerbork, Lovell, Nancay and Sardinia), seeking to use high-precision pulsar timing to directly detect GWs. Regular monitoring of high-precision millisecond pulsars by the EPTA has been the basis for the EPTA GW limit established [6]. However more precise timing of millisec- 


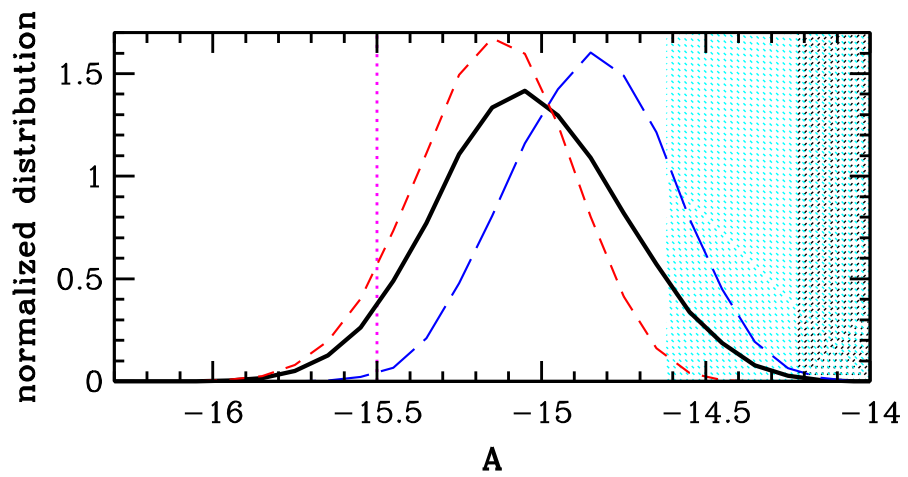

Fig. 1 Normalized distributions of the expected GW amplitude $A$ at $f=1$ year $^{-1}$. Black solid line, all models; green dot-dashed line, fiducial models only; red short-dashed line, models employing the double power law given by; blue long-dashed, models including SMBH measurements in BCGs. The shaded area marks the region excluded by the best current PTA limit [5], whereas the magenta dotted vertical line represents what can be achieved by timing 20 pulsars at $100 \mathrm{~ns}$ rms precision for 10 years

ond pulsars is necessary for GW detection. For this purpose, the Large European Array for Pulsars (LEAP) seeks to coherently add pulsar observations from the five 100-m class European telescopes to increase aperture and time-of-arrival accuracy. Combining the telescopes in a tied array creates a single telescope with the equivalent size of a 200-m dish, comparable in aperture to the illuminated Arecibo dish. Signals from different telescopes are corrected for time delays (due to differences in geometry, observatory clocks, instruments, atmospheric conditions, etc.), then added coherently to maximize signal-to-noise. LEAP observes 20-30 millisecond pulsars and associated phase calibrators on a monthly basis with a $128 \mathrm{MHz}$ bandwidth centered at $(\approx 1,400 \mathrm{MHz})$, simultaneously at all 5 telescopes. Recent progress includes the implementation of polarization calibration and RFI subtraction. Coherent addition of Westerbork and Effelsberg data leads to doubling of the signal-to-noise, as expected. Three and four-telescope addition with the Lovell and Nancay telescopes is currently being implemented. Additionally, the Sardinia Radio Telescope (SRT), a modern, fully-steerable $64 \mathrm{~m}$ dish with an active surface, joined LEAP observations in July 2013. LEAP observations are performed using a recently installed dual band LP $(300 / 1,400 \mathrm{MHz})$ receiver at the primary focus, and a FPGA-based $\mathrm{ROACH}$ backend for baseband recording. Currently one $16 \mathrm{MHz}$ sub-band can be recorded, while a computer cluster will soon permit the recording of the full $128 \mathrm{MHz}$ LEAP bandwidth. The SRT also has a Digital Filterbank backend, which, together with the ROACH, will allow the regular monitoring of millisecond pulsars for the EPTA.

\section{Searches for a stochastic gravitational wave background with pulsar timing arrays: a data analysis pipeline (Presenter: S. Chamberlin)}

Sources of a stochastic background are expected to exist and in the PTA frequency band include cosmic string cusps [7], relic GWs from inflation [8], first order phase 
transitions in the early universe [9], and (as the dominant source) SMBHBs $(M>$ $\left.10^{9} M_{\odot}\right)[1,10,11]$, which may occur when galaxies collide.

A GW, if present between the Earth and a pulsar, will perturb the pulses traveling from the pulsar [12] by inducing within them a redshift.For an array of multiple pulsars, it has been shown that there is a uniquely correlated perturbation in the pulsars' signals [13]. One method to search for GWs in PTA data is therefore to find an optimized cross correlation that takes into account the GW spectrum and noise power spectra.

In this talk, the optimal statistic for performing such a search was developed, based on an initial analysis by Anholm et al. [14] and utilized to construct a fully functional data analysis pipeline. The scaling laws that govern the signal-to-noise ratio for a PTA were discussed, and a software technique to determine frequentist upper limits was presented.

\section{Angular resolution and geometrical dependence of a pulsar timing array to detect individual gravitational-wave sources (Presenter: L. Wen)}

The most sensitive search for the GWB will come from an optimal combination of all available pulsar timing data. Wen presented analytical expressions for (1) the angular resolution of a PTA for individual GW sources; and (2) the dependence of a PTA's sensitivity to the two GW polarization on the geometrical distribution of pulsars.

The calculation of the angular resolution of a PTA is an extension from previous work for ground-based GW detectors [15] and recent work on PTAs [16]. Wen presented closed-form analytical expressions using Fisher Matrix formalism. Wen showed that when pulsars' distances are precisely known, the angular resolution follows something similar to the diffraction limit. Roughly speaking, the uncertainty in position (in solid angle) is inversely proportional to the weighted sum of the squares of projected areas formed from pulsar-pulsar and pulsar-Earth pairs in the plane perpendicular to the incoming GW direction. The weighting factors are on the order of $\left(f_{\mathrm{GW}}^{2} \mathrm{SNR}^{2}\right)^{2}$ for a $\mathrm{GW}$ of central frequency $f_{\mathrm{GW}}$ and signal-to-noise ratio SNR. Therefore, if the PTA consists of only two pulsars, the best angular resolution can be achieved from the direction perpendicular to the area formed by the two pulsars and Earth, and the worst angular resolution is from the direction parallel to the plane. However, if the pulsar distance is not known, the angular resolution is inversely proportional to $\mathrm{SNR}^{2}$. The Square Kilometre Array (SKA) will possibly offer the best distance determination for the millisecond pulsars within a PTA. At $0.1 \%$ precision, the distance determination will not be good enough in general for the PTA to reach the diffraction limit discussed above. However, a small fraction of the pulsars within certain "optimal" angles with the earth relative to a GW might contribute to several orders of magnitude improvement for the angular resolution.

Wen also showed that if we combine data from a PTA optimally for each sky direction following the maximum likelihood ratio principle, we can form new "twosignal" data streams representing two wave polarizations. The relative SNRs for these two signal streams can be shown to depend on the SNR for each pulsar and on the relative differences in polarisation angles between every two pulsars defined in the plane perpendicular to the $\mathrm{GW}$ direction. If there are $N_{p}$ pulsars, we should also have 
$N_{p}-2$ null streams in the sense that they have no response to GWs as discussed in Refs. [17-19]. These null streams can therefore be examined to verify or veto a GW trigger.

\section{Continuous gravitational wave search methods and results from PTAs (Presenter: J. A. Ellis)}

This presentation discussed the current sensitivity of PTAs to continuous GWs from SMBHBs. The time-domain posterior probability distribution, marginalized over pulsar timing parameters [6], was introduced for single source searches with PTAs and a Bayesian [20] and Frequentist [21] framework was reviewed. It was shown that it is indeed possible to use the unknown pulsar distance in the coherent bayesian search pipeline through the use of clever jump proposal distributions, thus making it possible to break the degeneracy between chirp mass and distance to the source. The angular sensitivity of current PTAs was then discussed and it was shown that the IPTA is crucial for the best all-sky sensitivity to GWs. With current IPTA datasets, we are approaching a sensitivity at which we have a $3 \%$ chance of detecting a single continuous GW, according to simulations done by Alberto Sesana, presented at this meeting. Finally, it was shown that the existence of an electromagnetic counterpart SMBHB can enhance our GW detection significance significantly if the period of the binary is measured electromagnetically.

\section{Characterizing gravitational wave stochastic background anisotropy with pulsar timing arrays (Presenter: C. M. F. Mingarelli)}

Detecting a stochastic GWB, particularly radiation from individually unresolvable SMBHBs, is one of the primary targets for PTAs [22-25] Increasingly more stringent upper limits are being set on these signals under the assumption that the background radiation is isotropic. However, some level of anisotropy may be present and the characterization of the GW energy density at different angular scales carries important information.

We define the level of anisotropy as the ratio of the standard deviation in the GW power emanating from a given solid angle to the expected power from that angle, $\sigma_{\mathrm{gw}} / \mu_{\mathrm{gw}}$. We find that the fractional level of anisotropy scales as

$$
\frac{\sigma_{\mathrm{gw}}(f)}{\mu_{\mathrm{gw}}(f)} \approx 0.2\left(\frac{f}{10^{-7} \mathrm{~Hz}}\right)^{11 / 6}\left(\frac{5 \mathrm{yr}}{T_{\mathrm{obs}}}\right)^{-1 / 2}\left(\frac{l}{2}\right)^{1 / 2} \alpha^{1 / 2},
$$

where $f$ is the frequency of the GW background, $T_{o b s}$ is the observation time, $l$ is the multipole moment index, $\alpha(y) \equiv\left(y^{2}+y+1\right) /(3 y)$ and $y=D_{M} / D_{m}$, where $D_{M}$ is the maximal distance at which sources can be located, which is set by cosmology and the history of SMBH formation. Meanwhile, the minimal distance $D_{m}$, corresponds to the maximal distance at which individual binaries can be resolved. 
We then show that the standard analysis for isotropic backgrounds can be generalized in a conceptually straightforward way to the case of generic anisotropic background radiation by decomposing the angular distribution of the GW energy density on the sky into multipole moments. We introduce the concept of generalized overlap reduction functions, $\Gamma_{l}^{m}$, which characterise the effect of the anisotropy multipoles on the correlation of the timing residuals from the pulsars timed by a PTA [26]. In a search for a signal characterized by a generic anisotropy, the generalized overlap reduction functions play the role of the so-called Hellings and Downs curve used for isotropic radiation [13].

We compute and present the generalized overlap reduction functions for a generic level of anisotropy and PTA configuration, and present new analytic overlap reduction functions for the dipole and quadrupole case.

A Bayesian analysis approach based on the formalism that we have presented here has now been developed by Taylor and Gair [27]. It is also important to note that some data analysis methods currently use "compression" algorithms to speed up the processing of the data [28]. As a result of this, the high frequency sensitivity is compromised. This is the frequency band where anisotropy is more significant, and therefore future development of data analysis techniques will need to take this into account.

\section{Searching for anisotropic gravitational-wave backgrounds using pulsar timing arrays (Presenter: S. R. Taylor)}

Although the nanohertz GW strain budget is comprised of many individual signals, and most likely constitutes a stochastic, isotropic background at low frequencies, the reality at higher frequencies is that the signal is completely dominated by only a handful of bright, nearby single-sources. As such, we cannot treat the signal as Gaussian and isotropic over the entire band [11,29]. Rather, we must have a robust formalism to describe continuous anisotropies in the energy-density of the GW background, and hotspots of GW-power from finiteness of the background signal. Hence, the current stochastic GW background search algorithms must be generalised.

Taylor presented a robust Bayesian data-analysis formalism to search for, and constrain, the level of anisotropy in a GW background. This implemented recent work [26] to generalise the GW-induced cross-correlation coefficients between pulsars, which, for an arbitrarily anisotropic background, are different from the standard Hellings and Downs isotropic-background coefficients. Although different, they are predictable, permitting a search to be carried out over the coefficients of a spherical-harmonic expansion of the GW energy-density's angular distribution, and for the Bayesian evidence to be computed for the purposes of anisotropic-versus-isotropic model selection. Through the development of this formalism, and tempo2 plugins to generate simulated datasets [30], Taylor showed that when we enter the precision science-era of PTAs, the GW background anisotropy must be incorporated into searches in order to fully characterise the signal. Via a systematic program of injection and recovery of signals containing varying levels of anisotropy, Taylor tested the robustness of their 
techniques, and illustrated the potential to recover sky-maps of the distribution of the GW background energy-density. For more details, see Ref. [27].

\section{Black hole hunting with pulsar timing arrays (Presenter: N. J. Cornish)}

This presentation reported on several projects carried out in collaboration with V. Corbin, A. Sesana and J. Romano, linked by the common theme that the signals from a population of SMBHBs may be better modelled as discrete point sources than as an isotropic, stochastic background. Using simulated timing data based on black hole population synthesis models, it was shown that the signals from such a population will be highly anisotropic [31] and non-Gaussian [29], though the non-Gaussianity may be difficult to discern in practice due to the limited time-frequency volume of the data. Remarkably, the Hellings-Downs correlation pattern for pairs of pulsars was shown to hold for isolated, point sources, so analyses that assume isotropy will still be effective at detection, though with sub-optimal sensitivity [31]. The likely properties of the brightest individual sources were discussed, along with Bayesian estimates for how well the source parameters could be inferred from the data [32]. Using the so-called "pulsar term" in the analysis will allow the mass and distance to the binaries to be determined, but with an accuracy set by how well the frequency [32], not the phase $[33,34]$ of the pulsar term is measured.

\section{A new method to search for supermassive black hole binaries by (Presenter: P. Rosado)}

The goal of this work [35] is to take a step towards understanding the PTA signal by assigning galaxies from the Sloan Digital Sky Survey (SDSS, [36,37]) a probability of hosting a SMBHB. If the signal resembles a stochastic background, our method can give insight on its statistical properties (deviation from Gaussianity, anisotropy, etc.); on the other hand, our work can also reveal the strongest individual GW emitters and their spatial distribution. The position and mass of galaxies with largest probabilities of hosting a SMBHB can be used in targeted searches for single sources [20].

To construct the searching method we first study the properties of merging galaxies from a mock catalog produced in the Millennium Simulation [38,39]. We find that galaxies that recently suffered a major merger (these are the galaxies that might host SMBHBs) can be distinguished from non-merging galaxies by just observing their stellar mass and redshift. Using only this information, we perform a search on the mock catalog and check how well MBHB hosts are identified. The same search is carried out on the SDSS, and on a mock catalog adapted to the observational limitations of the SDSS; this allows us to test the efficiency of the method.

We also investigate the neighborhoods of hosts of MBHBs and find that their distribution of neighbors below $10 \mathrm{Mpc}$ can be distinguished from that of average galaxies. The efficiency of the search does, however, not improve by including this information. Nonetheless, the study of galaxy neighborhoods allows us to conclude that the first detectable MBHBs will most likely be located in dense environments. 
The search is conducted with machine ;earning and Bayesian algorithms, with consistent outputs. The resulting skymap covers less than a fifth of the sky (which corresponds to the spectral coverage of the SDSS). Similar techniques can be used in the near future with larger and more complete catalogs to get a more accurate, observationally-based portrait of the true PTA signal. Moreover, the search technique can be combined with others [40] in order to remove false positives from the list of candidates.

\section{The Parkes pulsar timing array project (Presenter: R. M. Shannon)}

The Parkes Pulsar Timing Array (PPTA) project monitors $20 \mathrm{~ms}$ pulsars with the 64-m Parkes radio telescope with the primary goal of detecting gravitational radiation and other signals correlated amongst the pulsars. In this presentation, Shannon highlighted recent results of the project.

Shannon gave an overview of the project and its first data release [22]. This data release contained two datasets from the PPTA project: one dataset (DR1) that was optimally corrected for the effects of interstellar dispersion measure (DM) variations and a second, extended, dataset (DR1E) that included archival single-band observations for which DM correction was not possible.

One correlated signal that may be present in pulsar timing datasets is associated with variations in time standards. Variations in a time standard induce a common correlated signal in all pulsar timing observations. Shannon presented the results of a search for time variations in the PPTA DR1E dataset [41].

Shannon also presented a new limit on the strength of the GWB derived from the PPTA DR1E dataset. The limit was determined by conservatively comparing a detection statistic formed from PPTA observations to simulated datasets. This approach enables limit to be set for backgrounds that followed both Gaussian and non-Gaussian statistics [42]. sFor a Gaussian background with a strain spectrum $h_{c}(f)$ of the form $h_{c}(f)=A f^{-2 / 3}$ (see Eq. 1) the background was limited to be $A<2.4 \times 10^{-15}$ with $95 \%$ confidence. Shannon interpreted the limit in the context of three models of the GWB produced by SMBHBs [2,29,42]. The models were ruled out with between $\approx 50$ and $90 \%$ probability, showing that pulsar timing measurements were sensitive to backgrounds of astrophysical relevance [5].

Acknowledgments LW acknowledges funding support from Australian Research Council.

\section{References}

1. Sesana, A., Vecchio, A., Colacino, C.N.: The stochastic gravitational-wave background from massive black hole binary systems: implications for observations with pulsar timing arrays. MNRAS 390, 192 (2008)

2. Sesana, A.: Systematic investigation of the expected gravitational wave signal from supermassive black hole binaries in the pulsar timing band. MNRAS 433, 1 (2013)

3. McConnell, N.J., Ma, C.-P.: Revisiting the scaling relations of black hole masses and host galaxy properties. Astrophys. J. 764, 184 (2013)

4. Hlavacek-Larrondo, J., et al.: On the hunt for ultramassive black holes in brightest cluster galaxies. MNRAS 424, 224 (2012) 
5. Shannon, R.M., et al.: Gravitational-wave limits from pulsar timing constrain supermassive black hole evolution. Science 342, 334 (2013)

6. van Haasteren, R., Levin, Y., McDonald, P., Lu, T.: On measuring the gravitational-wave background using pulsar timing arrays. MNRAS 395, 1005 (2009)

7. Ölmez, S., Mandic, V., Siemens, X.: Gravitational-wave stochastic background from kinks and cusps on cosmic strings. Phys. Rev. D 81, 104028 (2010)

8. Starobinskiř, A.A.: Spectrum of relict gravitational radiation and the early state of the universe. Sov. J. Exp. Theor. Phys. Lett. 30, 682 (1979)

9. Caprini, C., Durrer, R., Siemens, X.: Detection of gravitational waves from the QCD phase transition with pulsar timing arrays. Phys. Rev. D 82, 063511 (2010)

10. Jaffe, A.H., Backer, D.C.: Gravitational waves probe the coalescence rate of massive black hole binaries. Astrophys. J. 583, 616 (2003)

11. Sesana, A., Vecchio, A., Volonteri, M.: Gravitational waves from resolvable massive black hole binary systems and observations with pulsar timing arrays. MNRAS 394, 2255 (2009)

12. Detweiler, S.: Pulsar timing measurements and the search for gravitational waves. Astrophys. J. 234, 1100 (1979)

13. Hellings, R.W., Downs, G.S.: Upper limits on the isotropic gravitational radiation background from pulsar timing analysis. Astrophys. J. 265, L39 (1983)

14. Anholm, M., Ballmer, S., Creighton, J.D.E., Price, L.R., Siemens, X.: Optimal strategies for gravitational wave stochastic background searches in pulsar timing data. Phys. Rev. D 79, 084030 (2009)

15. Wen, L., Chen, Y.: Geometrical expression for the angular resolution of a network of gravitational-wave detectors. Phys. Rev. D 81, 082001 (2010)

16. Boyle, L., Pen, U.-L.: Pulsar timing arrays as imaging gravitational wave telescopes: angular resolution and source (de)confusion. Phys. Rev. D 86, 124028 (2012)

17. Wen, L., Schutz, B.F.: Coherent network detection of gravitational waves: the redundancy veto. Class. Quantum Gravit. 22, 1321 (2005)

18. Chatterji, S., et al.: Coherent network analysis technique for discriminating gravitational-wave bursts from instrumental noise. Phys. Rev. D 74, 082005 (2006)

19. Wen, L.: Data analysis of gravitational waves using a network of detectors. Int. J. Mod. Phys. D 17, 1095 (2008)

20. Ellis, J.A.: A Bayesian analysis pipeline for continuous GW sources in the PTA band. Class. Quantum Gravit. 30, 224004 (2013)

21. Ellis, J.A., Siemens, X., Creighton, J.D.E.: Optimal strategies for continuous gravitational wave detection in pulsar timing arrays. Astrophys. J. 756, 175 (2012)

22. Manchester, R.N., et al.: The parkes pulsar timing array project. PASA 30, 17 (2013)

23. Ferdman, R.D., et al.: The European pulsar timing array: current efforts and a LEAP toward the future. Class. Quantum Gravit. 27, 084014 (2010)

24. Hobbs, G., et al.: The international pulsar timing array project: using pulsars as a gravitational wave detector. Class. Quantum Gravit 27, 084013 (2010)

25. Demorest, P.B., et al.: Limits on the stochastic gravitational wave background from the North American nanohertz observatory for gravitational waves. Astrophys. J. 762, 94 (2013)

26. Mingarelli, C.M.F., Sidery, T., Mandel, I., Vecchio, A.: Characterizing gravitational wave stochastic background anisotropy with pulsar timing arrays. Phys. Rev. D 88(8), 062005 (2013)

27. Taylor, S.R., Gair, J.R.: Searching for anisotropic gravitational-wave backgrounds using pulsar timing arrays. Phys. Rev. D 88(8), 084001 (2013)

28. van Haasteren, R.: Accelerating pulsar timing data analysis. Mon. Not. R. Astron. Soc. 429, 55-62 (2013)

29. Ravi, V., Wyithe, J.S.B., Hobbs, G., et al.: Does a "stochastic" background of gravitational waves exist in the pulsar timing band? Astrophys. J. 761, 84 (2012)

30. Hobbs, G.B., Edwards.: The Australia Telescope Compact Array (/ Parkes radio telescope / Mopra radio telescope / Long Baseline Array) is part of the Australia Telescope National Facility which is funded by the Commonwealth of Australia for operation as a National Facility managed by CSIRO. R. T., \& Manchester, R. N. 2006, MNRAS, 369, 655

31. Cornish, N.J., Sesana, A.: Pulsar timing array analysis for black hole backgrounds. Class. Quantum Gravit. 30, 224005 (2013)

32. Corbin, V., Cornish, N.J.: Pulsar timing array observations of massive black hole binaries arXiv:1008.1782 (2010) 
33. Deng, X., Finn, L.S.: Pulsar timing array observations of gravitational wave source timing parallax. MNRAS 414, 50 (2011)

34. Lee, K.J., et al.: Gravitational wave astronomy of single sources with a pulsar timing array. MNRAS 414, 3251 (2011)

35. Rosado, P.A., Sesana, A.: Targeting supermassive black hole binaries and gravitational wave sources for the pulsar timing array. MNRAS, arXiv:1311.0883 (2013, submitted to)

36. York, D.G., et al.: The sloan digital sky survey: technical summary. Astrophys. J. 120, 1579 (2000)

37. Abazajian, K., et al.: The seventh data release of the sloan digital sky survey. Astrophys. J. Suppl. 182, 543 (2009)

38. Springel, V., et al.: Simulations of the formation, evolution and clustering of galaxies and quasars. Nature 435, 629 (2005)

39. Guo, Q., et al.: From dwarf spheroidals to cD galaxies: simulating the galaxy population in a $\Lambda \mathrm{CDM}$ cosmology. MNRAS 413, 101 (2011)

40. Ellison, S.L., et al.: Galaxy pairs in the Sloan Digital Sky Survey- VIII. The observational properties of post-merger galaxies. MNRAS 435, 3627 (2013)

41. Hobbs, G., et al.: Development of a pulsar-based time-scale. MNRAS 427, 2780 (2012)

42. McWilliams, S.T., Ostriker, J.P., Pretorius, F.: Gravitational waves and stalled satellites from massive galaxy mergers at $z \leq 1$. arXiv:1211.5377 (2012) 